Rüdiger Görner

\title{
Poetik der Kritik - Ästhetik des Deutens
}

El pensamiento / revoloteando / entre estas palabras

Octavio Paz, Blanco ${ }^{l}$

\section{Formen der Kritik - ein Präliminarium}

Zu den komplexen Vorgaben kritischen Interpretierens von Texten gehört jene Friedrich Schlegels, die er in den Lyceum-Fragmenten formulierte: „Poesie kann nur durch Poesie kritisiert werden. Ein Kunsturteil, welches nicht selbst ein Kunstwerk ist [...], hat gar kein Bürgerrecht im Reiche der Kunst.“2 Schlegel lenkte damit das Augenmerk auf das kritisch interpretierende Verfahren an sich, das zu einer Urteilsbildung führt; diese ist wiederum mit abhängig von mitgedachten Geschmacksurteilen. Kritisches Verfahren meint dabei stets auch ein Offenlegen der Wertungskriterien und die Frage, wie ein bestimmtes Vorverständnis reflektiert wird, das in die Deutung eines Kunstgegenstands mit eingeht. Die Art nun, wie sich diese (Selbst-)Reflexion im kritischen Diskurs vorführt, kann zu dessen eigenem Kunstcharakter beitragen. Wie diese komplexen Voraussetzungen in die kritische Auslegung eines (Sprach-)Kunstwerks hineinspielen, sei Gegenstand der folgenden Überlegungen. Sie gehen von der These aus, dass die , Ästhetik des Deutens' die sinnlich-intellektuelle Folge kritischer Verfahrensweisen ist, die ihrerseits das ,Machen’ einer kritischen Betrachtung, also ihre poesis, spiegelt. In der literarischen Moderne wurde am ehesten Hugo von Hofmannsthal Schlegels Forderung nach einer kunstwerkhaften Präsentation von Kunstkritik gerecht, und das vor allem deswegen, weil er dessen Prinzip, Kritik auch in Form von „Unterhaltungen“ über zu kritisierende Schriften zu entwickeln, sie also auf dialogische Art zu interpretieren, übernahm. Vermittels der Form fiktiver Gespräche - ob über Gottfried Keller, Goethes

\footnotetext{
${ }^{1}$ In: Octavio Paz, Suche nach einer Mitte. Die großen Gedichte. Spanisch und Deutsch. Übersetzung Fritz Vogelsang. Nachwort Pere Gimferrer. Frankfurt am Main 1980, S. 85. ("Das Denken / das umherflattert / zwischen den Wörtern").

${ }^{2}$ In: Friedrich Schlegel, Kritische Ausgabe Bd. 2 (= KFSA 2). Hrsg. v. Ernst Behler unter Mitwirkung von Jean-Jacques Anstett und Hans Eichner. München/Paderborn/Wien/Zürich 1967 ff., S. 162 (Fragment Nr. 117).
} 
Tasso, den „Charakter im Roman und im Drama“, über Gedichte oder ,über ein neues Buch $^{\text {‘3 }}$ - hob Hofmannsthal den Erlebnischarakter des dezidiert poetischen Deutens hervor. Auch die gesprächsweisen Kritiken Arno Schmidts stehen noch in dieser Tradition. Einen späten Ausläufer dieser ästhetisch wie intellektuell ansprechenden Form von Kritik bietet Hans Magnus Enzensbergers „Fünf Unterhaltungen über ,Jacques le fataliste“" (2004), die mit der überraschenden Bemerkung einsetzen, man habe diesen „ersten modernen Roman“ dann zumindest „halb verstanden“, wenn ihn als ein „ungeheuerliches Quodlibet“، verstehe. ${ }^{4}$ Entsprechend besteht eine Besonderheit unseres Ansatzes darin, dass er Aussagen zur ,Poetik der Kritik' und des Verstehens als einem ästhetischen Akt, die von Theoretikern, Autoren und ihren fiktiven Personen getroffen werden, als grundsätzlich gleichwertige Beiträge zur Klärung dieser Thematik ansieht. Denn die Reflexion ästhetischer Phänomene und kritische Ausdeutung sprachkünstlerischer Gegenstände versteht sich hier als ein prinzipieller Vollzug von urteilendem Erläutern, das gewissermaßen seine jeweiligen ,Agenten' sucht und findet seien sie erdacht oder historisch, real'. Hinzu kommt, dass hier Vertreter einer ,poetischen Kritik' berücksichtigt werden, die das Kritische als Teil ihrer künstlerischen Produktion verstanden haben; das begründet auch die Bandbreite unserer Beispiele. Theorie der Kritik und ihr praktischer Vollzug als Mittel des Verstehens werden hier als eine Einheit gewertet, die ihrerseits Formen des Fiktiven, des Gesprächs etwa oder einer erfundenen Kunstkritik, generieren und den Charakter (kritischen) Verstehens veranschaulichend instrumentalisieren.

Das Interpretieren als diskursives Ereignis, das sich als poetische Kritik sieht, meint nach Walter Benjamin ,weniger die Beurteilung eines Werkes als vielmehr die Methode seiner Vollendung. "5 Überraschend kommentarlos stellt Benjamin jedoch diese These neben die Behauptung von Novalis, nach der nur „das Unvollständige begriffen“ werden könne.

\footnotetext{
${ }^{3}$ In: Hugo von Hofmannsthal, Gesammelte Werke in zehn Einzelbänden. Bd. VII: Erzählungen, Erfundene Gespräche und Briefe, Reisen. Hrsg. v. Bernd Schoeller in Beratung mit Rudolf Hirsch. Frankfurt am Main 1979, S. 481-543.

${ }^{4}$ In: Hans Magnus Enzensberger, Scharmützel und Scholien. Über Literatur. Hrsg. V. Rainer Barbey. Frankfurt am Main 2009, S. 462-478, hier: S. 462.

${ }^{5}$ Walter Benjamin, Der Begriff der Kunstkritik in der deutschen Romantik. In: Ders., Gesammelte Schriften Bd. I.1, hrsg. v. Rolf Tiedemann und Hermann Schweppenhäuser. Frankfurt am Main 1991, S. 7-122, hier: S. 69.
} 
Entsprechend müsse man sogar die Natur - gemeinhin doch ein Modellfall organischer Vollendung - ,als unvollständig setzen“, um sie begreifen zu können. ${ }^{6}$

Friedrich Schlegels These von der Kunstwerdung der Wissenschaft und dem

Wissenschaftlich-Werden der Kunst, der Vereinigung von Poesie und Philosophie setzt jedoch nicht voraus, dass damit primär die Frage nach der „Methode“ der Werkvollendung gemeint ist. Seine Rezensionen sowie die „Charakteristiken und Kritiken“ belegen eindrucksvoll, dass er gerade die Beurteilung von Werken suchte. Sie konnte im Extremfall durch einen Halbsatz erfolgen und damit durch eine betont fragmentarische Kritik, wenn er etwa zu Scotts „Elegien“ in Schillers Horen schreibt: „Gewöhnliche descriptive poetry. “7 Schlegel begann die fragmentarische Kritik wenn nicht zu kultivieren, so doch mit ihr zu experimentieren. Bei Schlegel liest sich dieses Verfahren dann so: „Elegie [von Matthison]. Über diese kann ich aus einer sonderbar scheinenden, aber sehr triftigen Ursache kein Urteil fällen: aus nichts wird nichts." Oder: „Eine Nachahmung der ersten Satire des Juvenal [in der Übersetzung von Voß]. Hie und da ein guter Einfall, ein glücklicher Ausdruck. Im ganzen nicht fröhlich, oder nicht leidenschaftlich genug. Und welche Verse!“*8

Bemerkenswert ist, dass Schiller diese Art von Stichwortkritik überhaupt in seinen Horen veröffentlicht hat. Wichtiger von unserem Standpunkt aus betrachtet: diese fragmentarischen Kritiken bedürfen ihrerseits der Interpretation, wenn man allein an den zweideutigen Ausruf „Und welche Verse!“‘ denkt, der sowohl positiv wie auch abwertend gemeint sein kann. Das Nichts oder Nichtige der Matthisonschen „Elegie“ lässt dagegen über das Urteil keinen Zweifel. Die zu kritisierenden Texte fand Schlegel als geradezu emphatisch, vollendete' Dichtungen vor; doch antwortete er ihnen mit einer nicht minder emphatisch fragmentarischen Kritik. Was diese Art des Urteilens in jedem Fall leistet: Sie zieht den Leser in das Beurteilen hinein; die Unvollständigkeit bewirkt, dass er den Eindruck gewinnt, am knappen Urteilen teilzuhaben. Zugleich liefert sich der Kritiker Stichworte, die etwas von Bausteinen für eine ausführlichere Kritik haben. Schlegel bietet somit Abbreviaturen des Beurteilungsprozesses.

\footnotetext{
${ }^{6}$ Ebd., S. 69 f.

${ }^{7}$ In: Friedrich Schlegel, a.a.O., S. 41.

${ }^{8}$ Ebd., S. 20.
} 
Beim schriftlichen Sprechen über Literatur kommt hinzu, dass das fiktive Gespräch und der in ihm stattfindende Prozess des kritischen Urteilens ineinander übergehen, wobei sich der Leser wiederum eingeladen fühlt, sich in das Gespräch und damit in die Deutung von Dichtungen einzubringen. Dadurch entsteht ein doppelter Erlebniseffekt: das fiktive Gespräch über Literatur, das seinerseits Literaturkritik literarisiert sowie die diskursive Aufschlüsselung und Deutung des buchstäblich in Rede stehenden Werkes und seiner Teile werden eben durch die Gesprächsform unmittelbar nachvollziehbar und damit zu einem Musterbeispiel für die eingangs behauptete Wechselbeziehung vom Herstellen der Kritik und ästhetischer Erfahrung beim Deuten des literarischen Gegenstandes. Die implizite ironische Pointe ist dabei, dass das literarische Gespräch als Literatur seinerseits Gegenstand der Betrachtung sein kann, wodurch das literaturkritische Gespräch selbstreferentiell würde: Die fiktiven Teilnehmer des fiktiven Gespräch unterhalten sich über ihre Kriterien und Voraussetzungen ihres Urteilens und erlauben den ,lesenden Zuhörern' auf diese Weise eine ästhetische Erfahrung. ${ }^{9}$

Interpretieren bedeutet, den in Rede stehenden Gegenstand auszulegen, seine Kontexte erhellen, um ihn dadurch zu verlebendigen, motivische Konstellationen erkennen und in ihrer Bedeutung werten sowie stilistische Eigenheiten in ihrem Aussagewert erfassen. Auf diese Weise meint Interpretieren immer auch ein Vergegenwärtigen: ein historischer Text sieht sich in die aktuelle Interpretationszeit versetzt, wodurch sich auch das jeweilige Vorverständnis aktiviert und aktualisiert. Alles deutende Auslegen dient vorrangig dem Verstehen des Textes, seiner Genese und Struktur sowie der mit ihm verbundenen Intention, sofern sich dafür im Text oder in begleitenden Dokumenten (Briefen, Notizen, Tagebuchaufzeichnungen) Anhaltspunkte finden.

Texte sind Netze, voller Verknüpfungen und - doppeldeutig genug! - Maschen (im Sinne von ,durchlässigen Texturen“ und Hinweisen auf eine raffinierte Machart); als Beschreibungen legen sie sich über ein Objekt oder sie generieren fiktive Objekte, die mehr oder weniger abstrakt bis sinnlich sind. Die Wirkungsweise von Texten zu verstehen, die Art wie sie Stilmittel instrumentalisieren, ob sie assoziativ operieren oder

\footnotetext{
${ }^{9}$ Vgl. dazu: Rüdiger Görner, Ästhetische Idee - ästhetische Erfahrung. Überlegungen zu einem Wechselverhältnis im Umfeld der Ästhetik Kants. In: Athenäum 28(2018), S. 7388 .
} 
analytisch, spekulativ oder (schein-)logisch, ist das Hauptanliegen von Interpretation; sie muss sich ihrerseits Rechenschaft über ihr Erkenntnisinteresse geben. Ob es den Verstehensprozess wirklich kennzeichnet und ihm gar zuträglich ist, wenn er nahezu zwangsläufig ,,in ein Erfinden“ übergeht (,Jegliches Verstehen [...] erfindet Beziehungen, Bewegungen, Zusammenhänge, - Erfindungen, an denen die geistige Spannweite abzulesen ist."), wie Gerhart Baumann meinte ${ }^{10}$, erscheint zumindest fraglich. Zuzugeben ist aber, dass das Finden oder Auffinden von verstehensrelevanten Aspekten eines Textes oder Kunstgegenstandes zum ornamentierenden Erfinden verleiten kann.

Je komplexer die Erfahrungszusammenhänge sich uns darstellen, je weniger sogenannte gemeinsame Nenner in der informationsübersättigten Vielfalt unseres digitalen Zeitalters tragfähig sein und überzeugen können, je dringlicher artikuliert sich das Bedürfnis nach Verstehen. ${ }^{11}$ Im Wissenschaftsbetrieb lässt inzwischen nur allzu häufig die Fokussierung auf digitale Texteditionen das Interpretieren von Inhalten und Schreibformen verkümmern. ${ }^{12}$ Entsprechend ist es heute schon wieder viel, wenn eine Verständigung über Verstehensprobleme gelingt. Das wiederum verweist auf den engen Zusammenhang von Hermeneutik und Kommunikation. Die Leistung der Hermeneutik bleibt, von Anbeginn auf die „Sprachlichkeit jeglichen Verstehens“ gesetzt zu haben ${ }^{13}$ und damit auf die Kommunikationstauglichkeit hermeneutischer Befunde. Eine solche Kommunikation kann sich gerade auch auf einen spezifischen Aspekt beziehen, der für die diversen Varianten der ,Moderne' konstitutiv geworden ist: das Erfahren und Interpretieren von Ambivalenzen. In der Verwendung von Ambivalenzen äußert sich eine spezifische Art der Kritik, nämlich die einer Hinterfragung von vermeintlich eindeutigen

\footnotetext{
${ }^{10}$ Gerhart Baumann, Erfahren und Erfinden. Zweiseitige Erwiderungen. In: ders., Umwege und Erinnerungen. München 1984, S. 133-133, hier: S.113.

${ }^{11}$ Dazu grundlegend: Konrad Paul Lissmann, Theorie der Unbildung. Die Irrtümer der Wissensgesellschaft. Wien 2006. Vgl. dazu auch: Heike Ortner, Daniel Pfurtscheller, Michaela Rizzoli, Andreas Wiesinger (Hrsg.), Datenflut und Informationskanäle. Innsbruck 2014.

${ }^{12}$ Rüdiger Görner, Wenn die Saat des Digitalen aufgeht. Droht das Ende einer sinnvollen Textauslegung durch virtuelle Interpretationstechniken? In: Frankfurter Allgemeine Zeitung v. 27. Juni 2019, S. 6 (Seite ,Bildungswelten').

${ }^{13}$ Rüdiger Bubner, Die Karriere der Hermeneutik. Hans-Georg Gadamer zum neunzigsten Geburtstag. In: Neue Zürcher Zeitung v. 9. Februar 1990, S. 39.
} 
Gegegebenheiten. Das Ambivalente verdankt sich als Denk-und Ausdrucksfigur einem auf Wechselverhältnisse eingestellten Erkennen. Auch die Umkehrung gilt: Die Mehrdeutigkeit, sprich: semantische Mehrwertigkeit von Worten eignet sich für die sprachkünstlerische - und damit gezielte - Verwendung von Ambivalenzen.

Zweideutigkeiten dieser Art lassen sich bekanntlich kreieren, etwa durch Kennzeichnung und Verbindung von Bekanntem mit Fremdartigem. Exemplarisch spricht sich etwa in der poetischen, Zeitkritik enthaltenden Formel ,schwarze Milch der Frühe“ Paul Celans die Zweideutigkeit einer Leben spendenden Substanz aus; vergleichbar ambivalent wirken heute Hymnen an die Leben spendende Sonne auf uns - von Echnaton von Amarna bis Ingeborg Bachmann - lesen wir sie doch inzwischen auch unter den Wissens-Vorzeichen der gefahrvollen Erderwärmung und ihrer hautkrebserregenden Strahlenintensität. Das Verstehen des eigentlichen Sachverhalts und das seines ambivalenten Potenzials halten sich bei diesen Beispielen die Waage.

\section{Anschauung als Verstehen}

Im Zusammenhang einer Ästhetik des Verstehens gilt der zirkuläre hermeneutische Prozess an sich als ein ästhetisches Phänomen; dabei ist gleichfalls zu berücksichtigen, dass ein Verstehen denkbar ist, welches ausschließlich auf Anschauung und damit sinnlicher Erfahrung beruht. In der ,bloßen' Naturanschauung etwa hatte Goethe einen Weg gefunden, um seinem fundamentalen Misstrauen gegen den Schein abzuhelfen und Naturkunde gegen spekulative Ästhetik in Anschlag zu bringen. ${ }^{14}$ Goethe von Plotin geborgte Wort: „Wär' nicht das Auge sonnenhaft, / die Sonne könnt' es nie erblicken“15 findet sein ambivalentes Gegenstück in der Ankündigung des poetischen Ichs in Bachmanns Gedicht „An die Sonne“, es werde um ihretwegen „Klage führen über den

\footnotetext{
${ }^{14}$ Dazu grundlegend: Alfred Schmidt, Goethes herrlich leuchtende Natur. Philosophische Studie zur deutschen Spätaufklärung. München 1984.

${ }^{15}$ In: Johann Wolfgang von Goethe, Zur Farbenlehre. Didaktischer Teil. In: Werke. Hamburger Ausgabe Bd. 13: Naturwissenschaftliche Schriften I. Hrsg. v. Erich Trunz. München 1988, S. 324.
} 
unabwendbaren Verlust meiner Augen. “16 In der Beschreibung, also Versprachlichung der Art seines Verstehens der Farben und dem für sie zuständigen Wahrnehmungsorgan lieferte Goethe ein Beispiel für eine poetische Interpretation eines Naturphänomens, wenn er schreibt: „Das Auge hat sein Dasein dem Licht zu danken. Aus gleichgültigen tierischen Hülfsorganen ruft sich das Licht ein Organ hervor, das seinesgleichen werde, und so bildet sich das Auge am Lichte für Licht, damit das innere Licht dem äußeren entgegentrete. “17 Zweideutigkeiten scheinen hier nicht vorgesehen. Das natürliche Organ, das sich ,gebildet' hat, ist hier zu interpretierender Text-Ersatz. Goethe ,liest' das Auge in seiner Funktionalität und Idealität (Wechselverhältnis von Äußerem und Innerem), das freilich auf eine sprachlich kunstvolle Weise, womit er Schleiermachers Forderung entspricht, Hermeneutik immer auch als einen Kunstakt zu verstehen. ${ }^{18}$ Wolfgang Iser erläutert in seiner Abhandlung The Range of Interpretation die innere Verknüpfung von erkenntnispraktischen Aspekten des hermeneutischen Zirkels in diesem Zusammenhang: „The circle makes part and whole into shifting foils for one another, thereby converting grammar/psychology as well as divination/comparison into mutually mirroring viewpoints.“19

Verstehen ist auf Erkennbarkeit von Zeichen und Symbolen angewiesen. In Die Schrift und die Differenz hat Jacques Derrida jenen „Augenblick, wo ein Zeichen entsteht“ als einen Moment beginnender Wiederholung bezeichnet. ${ }^{20}$ Ohne Wiederholen könne das Zeichen nicht zum Zeichen werden. Ein solches ,Zeichen' als Graphem oder Hieroglyphe weist in die Richtung des Verstehens größerer Kontexte, in dem es steht. Zeichen sind demnach Zeiger oder Wegweiser in Richtung umfassenderen Interpretierens, ausgehend freilich von einem Detail, das sich etwa editionskritischen Einsichten verdankt

\footnotetext{
${ }^{16}$ In: Ingeborg Bachmann, Gedichte. Erzählungen. Hörspiel. Essays. 5. Aufl. München 2001, S. 62.

${ }^{17}$ Goethe, Farbenlehre, a.a.O., S. 323.

${ }^{18}$ Friedrich Schleiermacher, Hermeneutik und Kritik. Hrsg. v. Manfred Frank. Frankfurt am Main 1990, S. 91.

${ }^{19}$ Wolfgang Iser, The Range of Interpretation. New York 2000, S. 53. Iser rekurriert dabei - nicht unironisch - auf Emil Staiger, Die Kunst der Interpretation (1955). München 1971. Staigers theoretischer Beitrag zu dieser „Kunst“ bestand u.a. darin, die Kausalität als ästhetische Kategorie relativiert zu haben, an deren Stelle er die Forderung nach konsequenter, aber detailgenauer „Beschreibung“ ästhetischer Phänomene setzte. Ebd., S. 17.

${ }^{20}$ Jacques Derrida, Die Schrift und die Differenz. Übersetzt von Rodolphe Gasché. Frankfurt am Main 1976, S. 446.
} 
(Verwendung und Tilgung von Auslassungszeichen, Gedankenstrichen,

Verschreibungen, Umschreibungen etc.) oder lexikalischen Besonderheiten (bekannte Beispiele hierfür liefert ist das betont szientistische Vokabular und seine Umfunktionierung in Gedichten Gottfried Benns, Paul Celans, Thomas Klings, Ulrike Draesners oder Durs Grünbeins).

Doch kommen wir noch einmal, aber genauer, auf die Textkonstitution und ihre Implikationen für das Interpretieren zurück. Im Text entstellt sich zunächst die Unmittelbarkeit der ihm zugrunde liegenden sinnlichen Wahrnehmung, will besagen: Jede Verschriftlichung einer sinnlich unmittelbaren Erfahrung bedarf einer sprachlichen Leistung, die niemals identisch mit dem (nicht sprachlich) Erlebten sein kann, sondern ihrerseits eine spracheigentliche Unmittelbarkeit erzeugen muss. Alles Schreiben beginnt, so gesehen, mit einem Akt der Entfremdung. Der rhetorisch-stilistische Aufwand, der zu treiben ist, um einen Text entstehen zu lassen, kaschiert diese Entfremdung von der sinnlichen Unmittelbarkeit, die anspruchsvolle Texte jedoch wiederherzustellen und die Kritik nachzuvollziehen versuchen. ,Anspruchsvoll' kann dabei auch bedeuten: evokativ werden durch gezielt eingesetzte Sprachmittel. Novalis notiert im Allgemeinen Brouillon, dass wir ,alles Fremde nur durch Selbstfremdmachung - Selbstveränderung Selbstbeobachtung ${ }^{621}$ verstehen, das bedeutet: gerade auch das Verstehen eines fremden Text erfordert, ihn in einen Bezug zum eigenen Selbst zu stellen, ihn dabei aber gleichermaßen in seiner Fremdheit zu reflektieren. Novalis spricht übrigens von der Interpretation als einer „Erschöpfungsmethode“, was zweideutiger nicht sein kann: sie schöpft Bedeutungen und Zusammenhänge aus und gleichzeitig führt sie zur Ermüdung, wenn die Form der kritischen Vermittlung den Leser nicht anzuregen versteht und der Stil stumpf bleibt.

Und noch ein weiteres Wort zum Text: Er hat kartografische Eigenschaften; er bildet denksprachliche Landschaften ab, erlaubt die Verortung von Vorstellungen, Brüchen und Übergängen, verweist auf Unebenheiten im Schreibprozess, verhält sich im Wesentlichen kongruent zu den in den Blick genommenen Wirklichkeiten. Diese wiederum können wissenschaftlicher und fiktionaler Natur sein, Analyse und Imagination entstammen,

\footnotetext{
${ }^{21}$ Novalis, Werke, Tagebücher und Briefe Friedrich von Hardenbergs. Hrsg. v. Hans-Joachim
} Mähl und Richard Samuel. Bd. 2. Darmstadt 1999, S. 670. 
selbstgenerierend scheinen oder das jeweilige Zwischenergebnis von Verwandlungen sein. Jeder Text hat jedoch seine eigene ästhetische Wirklichkeit und Wahrheit, was freilich darauf verweist, dass beide Konstruktionen sind.

In Zeiten der „fake news“ hat jedoch Salman Rushie Schriftsteller, Journalisten und Philosophen dazu aufgerufen, aus den Trümmern der Wahrheit durch Texte „die Aufgabe des Wiederaufbaus zu übernehmen.“ Emphatisch fordert er: „Wir müssen den Glauben unserer Leser an die Realität und die Wahrheit wiederherstellen. Mit einer neuen Sprache, von Grund auf.“ Dieser Aufruf fußt auf Rushdies Einsicht, „,ass der Zusammenbruch des alten Realitätsverständnisses die bedeutende Realität geworden sei; und dass die Welt vielleicht am besten erklärt werden kann mit gegensätzlichen, meist unvereinbaren Narrativen. “22 Doch weil dem in der Nachmoderne unzweifelhaft so ist, wirkt der Rekurs auf einen stabil vorausgesezten Wahrheitsbegriff wenig überzeugend. Denn die - ästhetische - Wahrheit eines Textes ${ }^{23}$ liegt nun einmal in seiner Literarizität oder analytischen Beschreibungskapazität, sprich: in der Art, wie er Sprache behandelt und das Geschriebene und damit die Wirklichkeiten, die es schafft und/oder ,reflektiert', erleben lässt. Untersuchen wir literarische Texte, dann gilt unsere Aufmerksamkeit dieser textspezifischen Art der Sprachbehandlung und dem, was sie generiert. In der ästhetischen Wahrheit eines literarischen Textes wie jedes Kunstwerks überhaupt manifestiert sich ein ausschnitthaftes Deutungsangebot von ,Welt'.

Dabei ist der Unterschied zwischen einem interpretierenden und auslegenden Untersuchen von Texten kardinaler Natur. Interpretieren bedeutet: Dazwischentreten, deutend performativ werden. Das exegetische Auslegen ist deutlich philologischer konditioniert, wobei beides zur Selbstzurücknahme im Zweifelsfall tendieren sollte.

(Hier weitermachen!)

\footnotetext{
${ }^{22}$ Salman Rushdie, „Fake“. Vom Zustand der Wahrheit in Literaturgeschichte und Gegenwart. In: Süddeutsche Zeitung Nr. 114 v. 19.-21. Mai 2018, S. 17.

${ }^{23}$ Dazu ausführlich: Käthe Hamburger, Wahrheit und ästhetische Wahrheit. Stuttgart 1979. Vgl. auch: Odo Marquard, Aesthetica und Anaesthetica. Philosophische Überlegungen. Paderborn, München, Wien, Zürich 1989, S. 11-20.
} 
Nichts schadet dem Interpretieren und Auslegen als einer Kunst, denn sie ist eine, mehr, als die auftrumpfende Gebärde, die Deutungshoheit suggerieren will. Als Deutende bedürfen wir der Fähigkeit, uns an Texte anzunähern, sie approximierend zu taxieren, auszuloten in ihren Tiefen und Untiefen. Eine tentative Deutung ist Texten gemäßer als eine, die nur Eindeutigkeiten kennen will, die es schon deswegen nicht geben kann, weil die in Rede stehenden literarischen Texte ihrerseits nicht mehr sein können als sprachliche Annäherungen an ein Phänomen. Annäherung nicht Aneignung sei daher die Devise der Textauslegung, der es um Bedeutungsöffnung zu tun sein sollte, nicht um Dogmatismus und bloßen Schematismus im Deutungsverfahren.

\section{Kritisches Erfassen - mit Lyrischem bei Nietzsche}

Literarische Texte unterhalten, auch wenn sie noch so sprachgenau erscheinen, zwangsläufig ein Unschärfe-Verhältnis zu den Dingen und Kontexten. Es gilt - entgegen der Auffassung des Erzählers in Rilkes Malte Laurids Brigge - ein Verhältnis aufzubauen gerade gegenüber dem Ungefähren. Interpretierend oder auslegend sind wir allemal Relationisten. Das ist kein Ausdruck von Sprachskepsis, sondern nur ein nüchterner Befund dessen, was Sprache ,wirklich' vermag und worauf sich die Kritik einzustellen hat. Dass es sich dabei keineswegs nur um eine deutsch geprägte Einsicht handelt, belegt der Erzähler in Benjamin Constants Roman Adolphe, veröffentlicht im Jahr 1816. Er berichtet von einem ,inneren Leiden“, das ,,alles, was wir zu sagen suchen, auf der Zunge entstellt und uns einzig erlaubt, uns in vagen Worten auszudrücken oder mit einer mehr oder minder bitteren Ironie, als wollten wir uns an unseren Gefühlen rächen für den Schmerz, den wir empfinden, da wir diese nicht mitzuteilen vermögen. “24 Deuten und Auslegen bedeutet daher zunächst einmal, sich Einlassen-Können auf das Ungewisse und Unbestimmte in Texten und das heißt: diesen Zustand erleben können. Dabei gilt es sich zu vergegenwärtigen, dass ihre Autoren oft erst nach langem Warten auf bestimmte Zeichen diese Texte verfasst haben, wobei das Warten auf Zeichen sich mit plötzlichen Einfällen kreuzen kann. Man ahnt es, hier ist von Nietzsche die Rede,

\footnotetext{
${ }^{24}$ Benjamin Constant, Adolphe. Anekdote. Gefunden in den Papieren eines Unbekannten. Aus dem Französischen übersetzt von Eveline Passet. Nachwort von Manfred Gsteiger. Zürich 1998, S. 27.
} 
dessen lyrisches Werk erst in letzter Zeit jene Würdigung erfährt, die es verdient. ${ }^{25}$ Sein Fall ist für unsere Diskussion deswegen so besonders, weil er aus Gedichten über Denkund Anschauungserlebnisse besteht und aus solchen, die den Interpretationsvorgang selbst als erlebnishaft thematisieren und parodieren. Die deutschsprachige Lyrik hat nur in Gottfried Benn einen vergleichbaren Fall aufzuweisen, der freilich ohne die Vorlage Nietzsches so nicht vorstellbar wäre, wobei Nietzsche anders als Benn am dialogischen Prinzip im Dichten festhielt. Gedichte wie „Sils Maria.“, „Narr in Verzweiflung.“ und vor allem „Nach neuen Meeren.“ zeigen, worum es Nietzsche ging: das Vereindeutigen von Deutungen zu unterlaufen. Das poetische Ich wird darin zum Narren und Kritiker zugleich wie zum alles auslöschenden „Wassermann“. Was Nietzsche hier vorführte, wirkte in seinem Anspruch radikaler als selbst sein stilistisches Vorbild Heine. Um das zu erreichen, begab sich Nietzsche sogar auf die Suche nach einem „,neuen Yorick“ als einem Mitstreiter, den er anreden konnte wie weiland Hamlet dessen Schädel in seinem Friedhofsmonolog. Dieser neue Yorick ist zudem auch ein neuer Columbus, dessen Bekenntnis in der endgültigen Fassung von 1887 das drittletzte unter den Liedern des Prinzen Vogelfrei darstellt, „Nach neuen Meeren.“, Teil des Ausklingens der Fröhlichen Wissenschaft, die ihrerseits poetische Auslegungswissenschaft in aphoristischer Form vorführt:

Dorthin - will ich; und ich traue Mir fortan und meinem Griff. Offen liegt das Meer, in's Blaue Treibt mein Genueser Schiff.

Alles glänzt mir neu und neuer, Mittag schläft auf Raum und Zeit -:

Nur d e i n Auge - ungeheuer Blickt mich's an, Unendlichkeit! ${ }^{26}$

\footnotetext{
${ }^{25}$ Vgl. die beiden wegweisenden Forschungsbände von Katharina Grätz, Sebastian Kaufmann (Hrsg.), Nietzsche als Dichter. Lyrik - Poetologie - Rezeption. Berlin/Boston 2017 sowie Christian Benne / Claus Zittel (Hrsg.), Nietzsche und die Lyrik. Ein Kompendium. Stuttgart 2017. ${ }^{26}$ Friedrich Nietzsche, Sämtliche Werke. Kritische Studienausgabe Hrsg.v. Giorgio Colli und Mazzino Montinari. Bd. 3 (= KSA 3) München 1988, S. 649. Vgl. dazu die jüngste textgenetische Auslegung von Werner Stegmaier, Im Auge der Unendlichkeit. Die Häutungen von Nietzsches
} 
Das Unabsehbare des Meeres verhindert genaues Sehen und damit Deuten. Zwar sieht das poetische Ich; alles scheint ihm ,neu und neuer“, aber vor allem wird es angesehen, und zwar durch das Auge der Unendlichkeit. In der ersten Strophe glaubt das Ich zunächst, seine Fahrt im Griff zu haben und damit über topographisch-nautische Deutungshoheit zu verfügen. Doch schon in den beiden folgenden Zeilen („Offen liegt das Meer, in's Blaue / Treibt mein Genueser Schiff") zeigt sich das Unbestimmbare. Man beachte den Punkt, den Nietzsche, ungewöhnlich genug, ans Ende seiner Gedichtüberschriften setzte, als wollte er das Thesenhaft-Bestimmte gegen seine Auflösung im Gedicht abgrenzen und damit sichern. Überhaupt, der auf Zeichen wartende lyrische Denker handhabte die Interpunktion virtuos, was wiederum den Musiker in ihm verriet, der nun einmal aus der Vielfalt partiturfähiger Zeichen zu schöpfen gelernt hat.

Zuweilen gewinnt man den Eindruck, als wollten Nietzsches Gedichte deutungsresistent werden und dabei philosophische Kontextbewusstheit ihrer Leserschaft suggerieren. Dabei gibt es Gedichte Nietzsches, die ihren Kontext explizit mitliefern. Zu bedenken wäre etwas ,Sils-Maria.“

Hier saß ich, wartend, wartend, - doch auf Nichts, Jenseits von Gut und Böse, bald des Lichts Genießend, bald des Schattens, ganz nur Spiel, Ganz See, ganz Mittag, ganz Zeit ohne Ziel.

Da, plötzlich, Freundin! wurde Eins zu Zwei - Und Zarathustra ging an mir vorbei . . ${ }^{27}$

Ganz nur Spiel: In Ecce homo wird Nietzsche befinden: „Ich kenne keine andre Art, mit grossen Aufgaben zu verkehren als das Spiel.“ Dass Interpretieren Spielcharakter haben muss, ist unter Musikern eine Selbstverständlichkeit. Philologen neigen dagegen oft dazu,

Gedicht ,Nach neuen Meeren'. In: Benne/Zittel (Hrsg.), a.a.O., S. 254-268 sowie Marie Wokalek, Nietzsches „Nach neuen Meeren“- Verdichtung eines Aufbruches ins Unbestimmte. In: Ebd., S. 269-279.

${ }^{27}$ KSA 3, 649. 
Versfüße allzu bleiern zu deuten. Um dem zuvorzukommen, ließ Nietzsche seine Fröhliche Wissenschaft mit einem Tanzlied „An den Mistral.“ enden. Um Denkerlebnisse oder literarische Texte in ihrer Erlebnishaftigkeit angemessen zu erfassen, bedarf es nun einmal einer deutenden Choreographie und einer choreographischen Deutung. Das bedeutet, wer Literatur auslegt oder interpretiert, muss einen Sinn haben für die Eigenbewegung der Worte und ihrer Bedeutungen - gerade in unvermuteten Kontexten, die ja erst das „,neu und neuer“ in der Erfahrung ermöglichen.

In der Deutungsgeschichte dieses Gedichts, die von Karl Löwith bis zu Manfred Riedel, Hans-Georg Gadamer, Günter Figal und Helmut Henne reicht, ${ }^{28}$ besteht ein Konsens, dass „Sils-Maria.“ eine Lyrisierung des ewigen Wiederkunftsgedankens darstelle, wobei sich daran exemplarisch erörtern lasse, inwiefern dies ein ,philosophisches' Gedicht sei, dem man sich nur mit einem entsprechenden Vorwissen nähern könne. Einzig Gadamer bot eine alternative - jedoch weniger überzeugende - Lesart, indem er „Sils-Maria.“, das ursprünglich den Titel „Portofino“ trug, im Kontext des „Willens zur Macht“ und nicht des Wiederkunftgedankens zu deuten unternahm. ${ }^{29}$ Handelt aber dieses Gedicht nicht eher von der Macht erlebender Wahrnehmung? Verträgt sich ,Wiederkunft' mit der im Gedicht prononciert vorgetragenen Plötzlichkeit? Steht hier nicht die entfunktionalisierte Zeit und Natur in Rede - mit Zarathustra als einem buchstäblich vorübergehenden Gast?

Für die Frage nach dem ,Nutzen und Nachteil' der Interpretation für das Erleben von Literatur ist eine weitere Frage konstitutiv: Wann, wo und wie beginnt und endet Interpretation? Im Sinne des hermeneutischen Verstehens und seiner philologischen Grundlegung ja wohl dort, wo es um Einsichten in das sprachkünstlerische Produktionsverfahren geht. Man kann diese Art des kritischen Auslegens ein Ausloten der poetischen Verfahrensweise nennen, aus der sich dann historische, biografische und soziokulturelle sowie kontextuelle Bedeutungsschichten ergeben, die ihrerseits philologisch und kulturpoetologisch freizulegen sind. Interpretation endet - und hier kommt wieder das von Novalis geprägte Wort „Erschöpfungsmethode“ zu seinem Recht

\footnotetext{
${ }^{28}$ Vgl. Katharina Grätz, Portofino in der Schweiz? Textgenese und Deutungsperspektiven von Nietzsches Gedicht „Sils-Maria“. In: Benne/Zittel (Hrsg.), Nietzsche und die Lyrik, a.a.O., S. 283-298, bes. S. $286 \mathrm{f}$.

${ }^{29}$ Hans-Georg Gadamer, Zwischen Wort und Begriff. Nietzsche und die Metaphysik. In: Manfred Riedel (Hrsg.), „Jedes Wort ist ein Vorurteil“. Philologie und Philosophie in Nietzsches Denken. Köln/Weimar 1999, S. 15-23.
} 
-, wenn wir einen Text vor unserem Zeit- und Verständniskontext für einstweilen ausgeschöpft halten. Wenn Gadamer nun das „Sils-Maria.“- Gedicht vor dem Hintergrund des „Willens zur Macht“ deutet, wofür es keine philologischen Anhaltspunkte gibt, dann handelt es sich hierbei um ein assoziatives Verstehen, das den üblichen Deutungskontext geradezu emphatisch überschreitet. Festzuhalten aber ist, dem Verstehensprozess selbst, dem Auslegen und Ausdeuten eignet Erlebnischarakter. Die kritische Interpretation von Literatur ist ihrerseits ein Erleben, das nur dann zum Lesevergnügen als gewöhnlicher Form der Wahrnehmung von Literatur querliegt, wenn dieses Interpretieren in einer Sprache geschieht, die sich nur noch in abstrakter Begrifflichkeit gefällt. Hier verläuft der entscheidende Bruch zwischen Wissenschaftlichkeit und Literatur, nämlich in einem Wahrnehmungsverhalten gegenüber Literatur, das deren Lebendigkeit durch die nur abstrakte Art ihres Interpretierens ignoriert.

Kritische Elementaritäten als ästhetische Erfahrung (nebst einem Hinweis auf Henry Home Lord Kame)

Dass Kritik auch Kunstwerk sein kann und werden soll, dieser höchste von Friedrich Schlegel ausgesprochene Anspruch, sollte nicht leichtfertig als ,unrealistisch' verworfen werden. Vielmehr ist diese Anforderung immer wieder neu zu begreifen als Aufgabe, die delikate Symbiose von Schöpferischem und Reflexion, Kunst und Kritik zu reflektieren und buchstäblich ,ins Werk` zu setzen. Der Ausgangspunkt hierfür ist weniger Kants Kritik der Urteilskraft als vielmehr die Elements of Criticism (1762-65) von Henry Home Lord Kame, die bereits 1765 in deutscher Übersetzung vorlagen ${ }^{30}$ und noch von Hegel in seiner „Einleitung in die Ästhetik“ zitiert wurde. ${ }^{31}$ Dieser heute im Vergleich zu James Ferguson und Adam Smith eher vernachlässigte Vertreter der schottischen Aufklärung ging in seinem zweibändigen Plädoyer für den Wert der Kritik von der sinnlichen Erfahrung aus, was ihn in die Nähe von Shaftesbury aber auch von Charles Batteux' Les

\footnotetext{
${ }^{30}$ Hier zitiert nach: Lord Kames Elements of Criticism. With the Author's Last Corrections and Additions. $7^{\text {th }}$ ed. Edinburgh/London 1788.

${ }^{31}$ Georg Wilhelm Friedrich Hegel, Vorlesungen über die Ästhetik. Erster und zweiter Teil. Mit einer Einführung hrsg. v. Rüdiger Bubner. Stuttgart 1977, S. 56.
} 
beaux arts réduits à un même principe (1746) brachte. Kame sprach von der „mixt nature“ der ,pleasures of the eye and the ear", die er den anderen sinnlichen Erfahrungen überordnete, wobei er ihnen einen ,middle place between organic and intellectual pleasures“ zuwies. ${ }^{32}$ Die organischen ,pleasures“ hielt Kame für kurzlebig, weil sie ihr „relish“ nach relativ kurzer Zeit einbüßten. Daher müssten gerade sie Gegenstand kritischer Analyse sein, um ihnen - man könnte sagen: Dauer durch Reflexion zu verschaffen. Worauf es Kame aber eigentlich ankommt, ist die Etablierung der Kritik als einer „rational science“, die in der Lage sei, unser Vergnügen an den schönen Künsten zu verdoppeln (,,redouble“) ${ }^{33}$ Sein Lob der Kritik liest sich wie folgt:

The science of rational criticism tends to improve the heart no less than the understanding. It tends, in the first place, to moderate the selfish affections: by sweetening and harmonizing the temper, it is a strong antidote tot he turbulence of passion, and violence of pursuit $[\ldots .]^{34}$

Indem die Kritik dazu verhilft, die Betrachtung von Kunst zu vertiefen, ihre Technik zu erfassen, bereichere sie, so Kame, unsere Kunsterfahrung und lasse die Kritik selbst zu einem Erfahrungwert werden, gespeist freilich von rationaler Einsicht in die Hervorbringungen der Kunst. Im Laufe seiner Untersuchungen über die Wirkungsweise stilistischer Kunstmittel stellte Kame „criticism“ entschieden neben „experience“. „Kritik‘ wird so als eine Erfahrungswissenschaft verstanden, die ihre Parameter jedoch offenzulegen hat. Das Gewinnen von kritischen Maßstäben gehört dabei auch zur Erfahrung.

Wirkliches Interpretieren geht über philologisches Erklären deutlich hinaus. Es nimmt Zusammenhänge auch dann in den Blick, wenn der Gegenstand der Interpretation einem einzelnen (sprachlichen) Kunstobjekt gilt. Als „,kritisches Verstehen“ setzt Interpretation Abstand und Empathie voraus; gleichzeitig bedarf es des Willens, das ,Andere` zu verstehen. Otto Friedrich Bollnow hat in Ableitung von Wilhelm Diltheys Verständnis

\footnotetext{
${ }^{32}$ Kame, Elements of Criticism, a.a.O., S. 3.

${ }^{33}$ Ebd., S. 7.

${ }^{34}$ Ebd., S. 9.
} 
von der Erlebnishaftigkeit des Deutens betont, dass sich aus dem „kritischen“ ein „schöpferisches Verstehen“ entwickeln könne, wobei er seinerseits ,kritisch“ nachfragte:

Wie kann das Verstehen als ein seinem Wesen nach rezeptiver Akt schöpferisch genannt werden? Aber die Verstehensleistung ist niemals eine bloße Wiederholung der verstandenen ursprünglichen Leistung, sondern stellt einen Vorgang völlig eigener Art dar, der seinem Wesen nach schöpferisch ist und seinen Gegenstand (sofern er nicht schon in sich selber zur unüberholbaren Vollkommenheit gebracht ist) produktiv fortzubilden gezwungen ist. ${ }^{35}$

Im Problem des Schöpferisch-Werdens des Verstehens schwingt die Forderung Friedrich Schlegels nach, Kritik müsse Kunst werden. Dabei wohnt dem produktiven Fortbilden des Interpretationsgegenstandes die Gefahr seiner Entstellung inne. Der Abstand zum Deutungsgegenstand wiederum enthält neben der kritischen auch die erste ästhetische Erfahrung, die der Interpretierende macht; muss er doch diesen Abstand als Differenz wahren und gleichzeitig überbrücken, ausloten und fruchtbar machen, als Abstand würdigen, indem er sich über diese Differenz Rechenschaft gibt. In diesem Abstand können kritisch-philologische Informationen oder Daten ihren Ort finden und zur Überbrückung eben dieser Differenz zum (sprachlichen) Kunstobjekt beitragen. Was aber ist an dieser Erfahrung ,ästhetisch"? Etwas Elementares: Das (kritisch) Gedeutete wird zum Sprechen gebracht, zu einem Sprechen über sich selbst, in das sich das Sprechen des Interpretierenden einbringt. So gesehen, ist Deutung immer mehr- oder zumindest zweistimmig.

Betont man, dass etwas ,wörtlich` zu verstehen sei, gar ,buchstäblich`, dann schränkt diese Vorgabe den Deutungsprozess ein. Steht dagegen der „Geist eines Phänomens, eines Worts“ im Mittelpunkt des interpretierenden Interesses, dann verbindet sich damit das Recht, „mit dem Bedeuten zu beginnen“ wogegen „,die Buchstäblichkeit die Weigerung ist, sich auf einen Bedeutungsprozeß einzulassen.“36

\footnotetext{
${ }^{35}$ In: Otto Friedrich Bollnow, Das Verstehen. Drei Aufsätze zur Theorie der Geisteswissenschaften. Mainz 1949, S. 56.

${ }^{36}$ Vgl. Roland Barthes, Erté oder An den Buchstaben. In: Ders., Der entgegenkommende und der stumpfe Sinn. Kritische Essays III. Aus dem Französischen von Dieter Hornig. 7. Aufl. Frankfurt am Main 2013, S. 122.
} 
Das Interpretieren von Kunst welcher Art auch immer bedeutet nicht, das Gedeutete auf einen Sinn festzulegen, wohl aber sich auf das zu Interpretierende in seiner Mehrschichtigkeit und Vielgestaltigkeit zu besinnen. Die Reflexivität des Besinnens verhindert die kanonisierende Vereindeutigung von Interpretationen, das ungefähr Widersinnigste, was Kunst durch den Akt der jeweiligen Interpretation widerfahren kann. Denn ein Sprachkunstwerk, das sich auf diese Weise vereindeutigen ließe, wäre ein bloßer Werbetext, der einen zielgerichteten und damit funktionablen Sinn benötigt und einzulösen hat. Ein wirkliches Gedicht dagegen, man kann es zergliedern nach allen Regeln der philologischen Kritik, entzieht sich der Vereindeutigung; allenfalls parodiert es sie. Man nehme etwa das dritte Gedicht aus Gottfried Benns Morgue-Zyklus (1912):

\section{Kreislauf}

Der einsame Backzahn einer Dirne, die unbekannt verstorben war, trug eine Goldplombe.

Die übrigen waren wie auf stille Verabredung ausgegangen.

Den schlug der Leichendiener sich heraus, versetzte ihn und ging für tanzen.

Denn, sagte er, nur Erde solle zur Erde werden. ${ }^{37}$

Die ,schöpferischste“ Deutung der Morgue-Gedichte hat Georg Baselitz durch seine Zeichnungen vorgelegt, ${ }^{38}$ indem er gerade das Kreisläufige im Verfall - als Sinnvorgabe des Gedichts - bildlich thematisierte. Bereits von der ersten Zeile geht eine drastische Konkretisierung dessen aus, was als Ausgangspunkt des „Kreislaufs“ zu bezeichnen wäre, wobei der vom Rhythmus her bedingten Verkürzung des Wortes „Backenzahn“ auf „Backzahn“ einer toten Dirne eine leicht ironische Qualität eignet. Der Backzahn mit seiner Goldplombe, dem eigentlichen Wertgegenstand, erweist sich als Hauptfigur des Gedichts; denn der „Leichendiener“ bleibt so anonym wie die „Dirne“. In vier knappen

\footnotetext{
${ }^{37}$ In: Gottfried Benn, Gedichte. Hrsg. v. Christoph Perels. Stuttgart 2012, S. 8.

${ }^{38}$ Gottfried Benn, Morgue und andere Gedichte. Mit Zeichnungen von Georg Baselitz. Stuttgart 2011.
} 
Zeilen erfahren wir jedoch Ausschnitthaftes über seinen Charakter. Er kennt keine Pietät, nur Funktionalität: Derer Gegenwert der leichengefledderten Goldplombe besteht in seinem kurzen Vergnügen. Das Gold gehört für ihn nicht zur „Erde“, weil es über einen materiellen Eigenwert verfügt, den er auskostet. Die gängige Aussegnungs-Wendung „Erde zu Erde, Asche zu Asche, Staub zu Staub“ qualifiziert das Gedicht mit einem „Nur“, was den natürlichen „Kreislauf“ angeht. Parallel geschaltet findet sich ein zweiter „Kreislauf“, nämlich der materielle: aus der „Goldplombe“ wird das Tanzvergnügen und dieses veranlasst den „Leichendiener“ zur Einsicht ins Kreisläufige überhaupt; denn zu erwarten steht, dass er sich beim Auffinden der nächsten Goldplombe ähnlich verhalten wird. Zudem spielt das Gedicht auf das Zweideutige des „Ausgehens“ an: Die „übrigen“ Zähne „waren wie auf stille Verabredung / ausgegangen“, ausgefallen oder eben verschwunden, wobei der Leichendiener selbst - quasi mit dem Erlös aus dem von seinem Wert gereinigten oder befreiten, jetzt „einsamen Backzahn“ - ,ausgeht“. Das Interpretieren von Gedichten erfordert, ausgehend von einem elementaren Sinn für das Deuten selbst, empfindendes Verstehen und damit auch Deutungsgespür. Die sinnliche Wirkung einzelner Wörter im vorliegenden Gedicht („Dirne“, „tanzen“, Erde“) überstrahlt ihre abstrakten Gegenstücke („Kreislauf“, „unbekannt“), ja sie überformt sie geradezu. Ungleich drastischer in der ersten Strophe des „Requiems“, dem Schlußstück der Morgue: „Auf jedem Tisch, zwei. Männer und Weiber / kreuzweis. Nah, nackt, und dennoch ohne Qual./ Den Schädel auf. Die Brust entzwei. Die Leiber / gebären nun ihr allerletztes Mal.“" ${ }^{39}$ Diese Reduktion des (toten) Seins auf das Physische kurz vor seinem Verfall zeigt Menschen als Opfer ihres Lebens. Der einzige abstrakte Begriff dieser Strophe ("Qual") sieht sich negiert. Jede der drei Strophen verfügt über ein einziges Abstraktum (,Sündenfall“ die zweite und schließlich „Rest“). Der „Sündenfall““ wird „begrinst“ von toten Mündern, und der „Rest“ der letzten Strophe gleicht einer Totnachgeburt.

In der „Morgue“ Benns scheinen die Worte selbst zu beobachten. Indem sie sagen können, was sie sehen, schockieren sie zwar, aber sie schwächen auch das Grauen über das Gesehene ab, beziehungsweise sie monopolisieren das grausige Erlebnis, werden zu ihm selbst. Das interpretierend zu zeigen ist eine ästhetische Erfahrung per se. Vom

\footnotetext{
${ }^{39}$ Benn, Gedichte, a.a.O., S. 9.
} 
Standunkt der Interpretation aus gesehen bietet zudem das Gedicht „Kreislauf“ ein poetologisch selbstreferentielles Beispiel für das hermeneutische, dem Zirkelschluss verpflichtete Verfahren: Das Interpretieren eines lyrisch thematisierten „Kreislaufs“ erfordert zwar keine in sich kreisende Argumentation, aber sie suggeriert sie zumindest. Um noch einmal auf Benns Gedicht „Requiem“ zu sprechen zu kommen, eine kritische Deutung müsste die Frage aufwerfen, ob Benn seine Strophen nicht mit krassen Bildern ,überfüllte' und sie damit eher um ihre Wirkung brachte, als dass sie diese verstärkt hätte. ,Schöpferisch“ bedeutet dann, durchaus zu zeigen, wie das Gedicht in entschlacktem Zustand aussähe. Zu behandeln wäre überdies die Art der Zeilenbrüche als Reimersatz mit ihren Folgen für die rhythmische Struktur des Gedichts.

Doch setzt die ästhetische Dimension des Interpretierens noch eine Spur tiefer an, nämlich bei der, wie Roland Barthes formulierte, analytischen Natur des Buchstabens, aber auch der Silben, etwa des viersilbigen Wortes „,ausgegangen“, dem das Gedicht Benns eine ganze Zeile einräumt, der beim Sprechen Wiegendes eignet, im Gegensatz zu dem eher staccato zu sprechenden „Denn, sagte er“ und dem geradezu ausgewogen klingenden Schlussvers: „nur Erde solle zur Erde werden“. Dass dieses Gedicht, das mit Verfallsmotiven beginnt auf einem „Werden“ endet, das aber selbst Ergebnis von Verfall ist, bestätigt das Kreisläufige dieser poetischen Aussage.

Was nun den Buchstaben selbst angeht, spricht Barthes von dessen Unschuld, und zwar wenn er ,alleinsteht“ und noch nicht zum Objekt von Aneinanderreihung geworden ist. ${ }^{40}$ Barthes riet zur Auflösung des (prekären) Wortes in seine buchstäblichen Bestandteile, um es zu entschärfen als dem ultimativen Akt dekonstruktivistischen Verstehens. Das widerspricht jedoch seiner vom Zweiten Korinther-Brief abgeleiteten These, nach der der Buchstabe töte, der Geist aber belebe. (2. Korinther 3, Vers 6) der Buchstabe hat eine alphabetische Bedeutung, aber für sich genommen keinen Sinn. Dieser wächst ihm erst durch die Aufreihung oder Verbindung mit anderen zu. Das hindert uns jedoch nicht daran, die symbolische Zeichenhaftigkeit von Buchstaben zu erwägen. Eine ,Poetik der Kritik` müsste hier ebenso ansetzen wie eine , Ästhetik des Deutens', nämlich dort, wo wieder ein Sinn dafür entsteht, dass der Buchstabe mehr ist als eine von der Linguistik konstatierte bloße Transkription eines bestimmten Lauts oder Mitlauts. Der Buchstabe ist

\footnotetext{
${ }^{40}$ Barthes, Der entgegenkommende und der stumpfe Sinn, a.a.O., S. 125.
} 
- von seiner ursprünglich magischen Funktion als Rune abgesehen - als graphisches Phänomen ein Zeichenkonvergat, in dem sich Symbolisches und Lautliches trifft - und das als kalligraphische Linie, wovon die Barocklyrik Zureichendes wusste, wenn sie in der Regel die ersten Buchstaben eines Gedichts als Majuskeln hervorhob. Der Schriftkult im George-Kreis übertrieb dieses Wissen dann jedoch bis zur (Selbst-)Parodie. Im Buchstaben scheint der Gegensatz zwischen Gegenständlichem und Abstraktem nichtig zu sein. Barthes folgert: „Denn ein Buchstabe sagt etwas aus und zugleich sagt er nichts aus, er imitiert nicht und symbolisiert dennoch, verabschiedet gleichzeitig das Alibi des Realismus und das des Ästhetizismus. “41 Wie kann der Buchstabe das? Nicht indem er seine vermeintliche „Unschuld“ ins Spiel bringt, wie Barthes etwas zu ursprungsverliebt meinte, sondern indem er als etwas Einzelnes in der jeweiligen Sprache und ihrem Schriftsystem gesetzt ist. Der Buchstabe repräsentiert weniger, als dass er ist. Deswegen freilich wirkt er trotz seiner ihm zugeschriebenen Symbolik vergleichsweise deutungsresistent. Doch meint Interpretieren immer auch, sich dieser lettristischen Bestandteile eines Sprachkunstwerks bewusst zu bleiben; Vergleichbares trifft für einzelne Noten, Farbtupfer, Striche, Bildsegmente eines Films zu. Denn wie das Einzelne wirkt, bedingt auch die Gesamtwirkung eines - und sei es fragmentarischen - Werks. Was der Buchstabe dennoch ,darstellt", wofür er ,steht"? Als ein Einzelner für das Vereinzelte oder Vereinsamen inmitten der Texte, aber auch für die Selbstbehauptung eines Zeichens, das nur auf sich selbst verweist.

\section{Erkenntnistheorie als ästhetische Praxis}

Der Sprung von dieser Mikroebene des Interpretierens zu dem Befund, mit dem der frühe Adorno die Einleitung zu seiner Metakritik der Erkenntnistheorie abschloss, ist zwar quantenhaft, aber unerlässlich, um sich einmal mehr den Zusammenhang unseres kritisch-deutenden Bemühens vor Augen zu führen. Adorno befindet:

Ist das Zeitalter der Interpretation der Welt vorüber und gilt es sie zu verändern, dann nimmt Philosophie Abschied, und im Abschied halten die Begriffe inne und werden zu Bildern. Möchte Philosophie als wissenschaftliche Semantik die

\footnotetext{
${ }^{41}$ Ebd., S. 123.
} 
Sprache in Logik übersetzen, so ist ihr als spekulativer noch übrig, die Logik zum Sprechen zu bringen. ${ }^{42}$

Der bildgewordene Begriff gleicht dem einen, aber erweiterten Buchstaben in den bedeutungswogenden Wortfeldern. Prüfen wir uns an dieser Stelle doch sogleich selbst. Wie kam es gerade eben zu dieser etwas pleonastischen Wendung ,bedeutungswogende Wortfelder"? Sie klingt ebenso sprachüppig wie manche der zuvor zitierten Verse von Benn, auch wenn ihre Funktion naturgemäß eine andere ist als die Häufung und Verdichtung drastischer Bildworte bei Benn. In einem rein diskursiv-kritischen Text fällt sie (für wohl viele unangenehm) auf. Das Bild vom „bedeutungswogenden Wortfeld“ stellte sich soeben ein, weil sich Texte in mir plötzlich als wogende Getreidefelder zeigten. Das ,Wortfeld‘ ist bekanntlich ohnedies ein linguistischer Fachbegriff als Bezeichnung für das Bedeutungsumfeld eines Wortes. Und so entstand das ,bedeutungswogende Wortfeld' nahezu folgerichtig. Das nun ist ein ästhetisch gewordener Kritikansatz, um analog auf den Begriff zu bringen, was Adorno in diesem Textsegment behauptet, das von einer marxistischen Prämisse ausgeht, dem Verändern der Welt nach dem Interpretieren ihres (Zeit-)Zustands. Anders als Marx, der keineswegs die Begriffe dadurch innehalten sah, sondern sie für mobilisiert hielt, verbindet der frühe Adorno mit dem Stornieren des Interpretierens zugunsten von deren handlungsorientierter Anwendung in der (politischen) Praxis, ein ästhetisches Moment, eben die Bildwerdung der Begrifflichkeit als dem Abschiedsgeschenk des philosophischen Denkens. Was es in seiner spekulativen Form noch leisten kann, ist die Versprachlichung der Logik, was die Bildlogik einschließt und überhaupt die Logik in der Kunst, die nicht nur kognitiven, sondern ästhetischen (Nicht-)Prinzipien folgt. Auch die Buchstaben-Logik des DADA würde dazu gehören; nicht minder die Eigenlogik des Komponierens im Zwölf-Ton-System.

Dass der Abschied seine eigene Ästhetik hat, in der ein kritisches Verstehen dessen enthalten ist, wovon man Abschied nimmt, hat Hans Ebeling als „Kritik der Moderne“

\footnotetext{
${ }^{42}$ Theodor W. Adorno, Gesammelte Schriften Bd 5: Zur Metakritik der Erkenntnistheorie. Drei Studien zu Hegel. Hrsg. v. Rolf Tiedemann unter Mitwirkung von Gretel Adorno, Susan BuckMorss und Klaus Schultz. Frankfurt am Main 2003, S. 47.
} 
thematisiert. ${ }^{43}$ Er sieht in ihr eine „Wahr-Nehmung“ und „Wahr-Setzung“ des Abschieds durch die Kunst und als „Kunst der Philosophie“ - mithin als Kunst des (kritischen) Denkens. ${ }^{44}$ Ebeling schreibt darin Paul Celans Dichtungen eine Schlüsselrolle zu, wenn es darum geht, Verständnis für einen seine Gegenwart behauptenden Abschied zu gewinnen. ${ }^{45}$ Es ist ein Abschied in der, wie man weiß, vergeblichen von Celan auf Heidegger gerichteten „Hoffnung, heute, auf eines Denkenden kommendes Wort im Herzen““ ${ }^{46}$ Celan selbst lieferte in seiner Meridian-Rede ein eindrucksvolles Beispiel für eine ästhetisch-poetologische Kritik, die ihrerseits Werkcharakter eignet und das Problem des Abschieds (von Traditionen) in der unbedingten Gegenwart der Rede auflöst. ${ }^{47}$ Auch Celans Notizen zum Vortragsprojekt „Von der Dunkelheit des Dichterischen“ belegen dieses kritische Moment, wobei hierfür nur ein solches Notat Textzeuge sein soll: „Im Gedicht ereignet sich etwas, passiert etwas: die Sprache als Dasein passiert die Enge dessen, der das Gedicht schreibt: sie geht hindurch und vorbei. " ${ }^{\text {"48 }}$ Celan redet von einer Sprache, die sich in die Enge begibt, im Gedicht eine „Engführung“ an sich selbst erlebt, eingehend, hindurchgehend, vorbeigehend - Letzteres im Wortsinn von ,passieren“, dem wiederum ein Abschiedsmoment innewohnt. Bei diesem Hindurch- oder Vorübergehen hinterlässt die Sprache jedoch Spuren, Wortspuren, aus denen das eigentliche Gedicht besteht. Diese Spuren gewinnen in ihm eine neue Form.

Thematisiert ist in dieser Textstelle Celans das kritische Verstehen eines poetischen Vorgangs als einem doppelten ästhetischen Prozess. Wenn wir Bollnows These folgen, vorgetragen in seiner Philosophie der Erkenntnis, dass ,,alles, was ich erkennen will, in gewisser Weise schon erkannt“ und Erkenntnis daher als „Korrektur und nähere Bestimmung des bisher unzureichend Erkannten“ zu verstehen sei, ${ }^{49}$ und wenn wir diese

\footnotetext{
${ }^{43}$ Hans Ebeling, Ästhetik des Abschieds. Kritik der Moderne. Freiburg i.Br./München 1989.

${ }^{44}$ Ebd., S. 9 f.

${ }^{45}$ Ebd., S. 120-129.

${ }^{46}$ Paul Celan, Gesammelte Werke in fünf Bänden. Bd. II. Hrsg. v. Beda Allemann u. Steffen Reichert. Frankfurt am Main 1986, S. 255.

${ }^{47}$ Paul Celan, Der Meridian. Endfassung - Entwürfe - Materialien. Hrsg. v. Bernhard Böschenstein und Heino Schmull. Frankfurt am Main 1999.

${ }^{48}$ Paul Celan, Mikrolithen sind, Steinchen. Die Prosa aus dem Nachlaß. Kritische Ausgabe. Hrsg. u. kommentiert v. Barbara Wiedemann und Bertrand Badiou. Frankfurt am Main 2005, S. 151. Hervorh. im Original.

${ }^{49}$ Otto Friedrich Bollnow, Philosophie der Erkenntnis. Erster Teil: Das Vorverständnis und die Erfahrung des Neuen. 2. Aufl. Stuttgart/Berlin/Köln/Mainz1970, S. 24.
} 
These auf Celans ästhetisch gewandeten kritischen Kommentar zu (s)einem poetischen Verfahren anwenden, ergibt sich dieser Befund: Was sich im Gedicht sprachlich ereignet, ,erkennen“ wir, weil wir mit der Sprache im Prinzip vertraut sind. Die „Korrektur“ besteht nun darin, die (poetische) Sprache in ihrem gedichtbedingt enggeführten Durchgangsstadium zu begreifen. Mit dieser „Korrektur“ ist zwar vorrangig Celans Lyrik gemeint, wobei er aber diesen - vermeintlichen - Sachverhalt verallgemeinern will. Celan spricht (und nicht nur hier) vom „Gedicht“ per se, von seiner eigenen poetischen Praxis ins Prinzipielle abstrahierend.

Die Ästhetik des Interpretierens ist ein kognitiver und sinnlicher Vorgang, dessen Qualität vom Zusammenspiel analytischer und poetischer Prozesse abhängt. Zum Primären dieser Art ästhetischer Erfahrung gehört die Kritik als Mittel des Erkennens. ${ }^{50}$ Entscheidend aber für die ästhetische Wirkung solcher Kritik ist deren sprachliche Gestalt, die den Deutungsvorgang transparent hält, sich am sprachlichen und allgemein künstlerischen Niveau des zu deutenden Werks orientiert, ihm zu entsprechend versucht, ohne es zu imitieren. (Das Parodieren kann bereits ein Aspekt schöpferischen Verstehens sein.) Kritisches Verstehen impliziert ein (Be-)Urteilen, dessen Maßstäbe wiederum als transparente Vorgaben zu verdeutlichen sind. Denn letztlich dient ästhetisches Deuten einem geistig-sinnlichen Zeigen, das ein nachvollziehbares Deutlich-Machen werkbedingter Phänomene leisten soll.

\footnotetext{
${ }^{50}$ Vgl. ebd, S. 100-102 („Die kritische Funktion der Erkenntnis”).
} 